\title{
DIMENSÕES DE UM SISTEMA DE APRENDIZAGEM EM AÇÃO PARA O ENSINO DE ADMINISTRAÇÃO
}

\author{
DIMENSIONS OF AN ACTION LEARNING SYSTEM FOR \\ HIGHER EDUCATION IN MANAGEMENT
}

Recebido em: 14/11/2011 Aprovado em: 26/01/2012

Avaliado pelo sistema double blind review

Editora Científica: Manolita Correia Lima

\section{ANIELSON BARBOSA DA SILVA anielson@pq.cnpq.br}

THALES BATISTA DE LIMA UNIVERSIDADE FEDERAL DA PARAÍBA

\author{
ANA LÚCIA BAGGIO SONAGLIO \\ CHRISTIANE KLEINÜBING GODOI \\ UNIVERSIDADE DO VALE DO ITAJAÍ
}

\section{RESUMO}

Este ensaio teórico objetiva delimitar as dimensões de um Sistema de Aprendizagem em Ação para o ensino de Administração. A proposição das dimensões do sistema surgiu da necessidade de introduzir um ambiente presencial ou virtual de aprendizagem, que auxilie docentes e discentes no processo de aprendizagem mais significativo, com recursos físicos e tecnológicos adequados ao contexto do mundo do trabalho contemporâneo. O sistema foi proposto com base em perspectivas teóricas da aprendizagem autodirecionada, da transformadora, da aprendizagem em ação e de sua contextualização para o campo de ensino superior em gestão. O sistema de aprendizagem em ação abrange cinco dimensões articuladas: I) o ambiente de aprendizagem do ensino em administração, 2) os estilos de aprendizagem dos professores e alunos, 3) a experiência docente e discente, 4) as estratégias de ensino em ação, e 5) a reflexão em ação. Considera-se que o sistema proposto, apesar dos desafios para a sua implantação no ensino de administração, pode aumentar as possibilidades de conexão entre a teoria difundida no contexto da formação acadêmica do administrador e a prática profissional no contexto das organizações, tornando o processo de aprendizagem mais significativo e transformador. Palavras-chave: aprendizagem em ação; educação gerencial; estilos de aprendizagem.

\section{ABSTRACT}

This paper aims to outline the theoretical foundations of an Action Learning System for the higher education field, specifically in management. The proposition of the foundations of the system has emerged from educational institutions' need to create a classroom and virtual environment of learning that help teachers and students in a more meaning ful learning process with physical and technological resources appropriate to the context of the contemporary labor market. The system was proposed based on the theoretical perspectives of self-directed learning, transformative learning and action learning and its context to the higher education field for management. The action learning system covers five connected dimensions: 1) the learning environment of management education, 2) teachers' and students' learning styles, 3) the teacher and student experience, 4) the teaching-in-action strategies, and, 5) reflection in action. Despite the challenges to implement the system in the teaching of management, it is considered that the proposed system has the capacity to increase the possibilities of connection between the theory provided in a management education and real-world, professional application within organizations, which contributes to a more meaningful and transformative learning process.

Keywords: action learning; management education; learning styles. 


\section{INTRODUÇÃO}

Os processos de aprendizagem em ação foram inicialmente institucionalizados no contexto das organizações e os estudos no ambiente educacional, que envolvem essa perspectiva, ainda são escassos. A possibilidade de transposição dos pressupostos da aprendizagem em ação, do ambiente da empresa para o ambiente da educação superior, é uma forma de articular o contexto da formação com o contexto da atuação profissional. No processo do ensino em administração, a aprendizagem em ação introduz a necessidade de o aluno aprender em tempo real e perceber a relevância do que aprende em suas vivências, para tornar a aprendizagem significativa e transformadora. Desta forma, é fundamental que o aluno esteja interessado em participar ativamente desse processo de aprendizagem.

As abordagens tradicionais de ensino são fundamentalmente objetivistas e balizadas por princípios que levam o aluno a uma resposta pré-determinada. É um processo passivo, uma vez que o conhecimento é considerado como "dado e absoluto" (BYRNE, 2002).

A aprendizagem de adultos deveria favorecer o autodirecionamento e o pensamento independente (CHIEN, 2004); envolver questões éticas e reflexivas, além de promover o comprometimento dos professores e a disposição dos alunos em direcionar as ações de seu aprendizado (MERRIAM; BROCKETT, 2007; FREIRE, 1979; 198I).

A aprendizagem em ação pode contribuir na melhoria do ensino superior em Administração, por meio da difusão de metodologias embasadas em uma perspectiva construtivista, que estimula os alunos ao pensamento crítico e à reflexão durante as discussões em sala de aula. Esse tipo de aprendizagem envolve a maior participação e interação dos alunos e os incentiva aos desafios (MARQUARDT, 2004).

Cerca de $13 \%$ do total de matrículas na educação superior, em 2oro, foram para o curso de Administração, o que revela a sua representatividade no sistema de educação superior brasileiro. Por outro lado, vivencia-se um contexto da formação de profissionais marcado pelo grande número de 
alunos por turma, pelo distanciamento entre teoria e prática, a dificuldade de vários docentes no planejamento e operacionalização de suas aulas em função da falta de qualificação e de tempo para difundir estratégias de ensino que estimulem a maior participação dos alunos, o maior engajamento nas atividades que demandam tempo para preparação e reflexão. Os processos de aprendizagem estão mais centrados numa aprendizagem mecânica do que em uma aprendizagem significativa na maioria dos cursos e instituições.

Percebe-se a necessidade de discutir o processo de aprendizagem dos futuros administradores e prepará-los para o mundo do trabalho, que requer dinamismo, criatividade, responsabilidade e a capacidade de pensar sistemicamente sobre os problemas organizacionais e da sociedade. Pensar sobre o ensino de administração, nos dias atuais, passa pela introdução de um sistema de aprendizagem que aproxime a teoria da prática e que torne as experiências vivenciadas pelos alunos significativas, por meio da difusão de estratégias de ensino mais coerentes com o contexto da atuação deste profissional no mundo do trabalho.

Este ensaio teórico pretende contribuir com esta problemática e objetiva delimitar as dimensões de um sistema de aprendizagem em ação para o ensino de Administração, e que foi desenvolvido para dar suporte teórico a um projeto criado por um grupo de pesquisadores brasileiros, vinculado ao edital Pro-Administração da capes. As dimensões propostas neste ensaio estão sendo pesquisadas por vários mestrandos e doutorandos, que pretendem, por meio de estudos empíricos, contribuir para elaboração de projetos pedagógicos mais dinâmicos e auxiliar os docentes a compreenderem a natureza dinâmica dos processos de ensino, principalmente porque os alunos possuem vários estilos de aprendizagem, que demandam a necessidade de utilizar estratégias que estimulem o pensamento reflexivo no contexto da ação, além de tornar o processo de aprendizagem mais significativo e tranformador.

As dimensões do sistema podem auxiliar gestores acadêmicos e professores na institucionalização de inovações no ambiente da aprendizagem, no conteúdo e nas estratégias de ensino difundidas nas universidades, centros universitérios e faculdades públicas e privadas. 
Para fundamentar a compreensão da articulação entre as dimensões do sistema de aprendizagem em ação, torna-se fundamental discutir perspectivas teóricas de aprendizagem que balizam a proposta do sistema, uma vez que o processo de formação de docentes na área de Administração não contempla a discussão sobre as teorias de aprendizagem de adultos.

O artigo também aborda as especificidades da aprendizagem em ação no contexto da administração para, posteriormente, apresentar o sistema e suas dimensões constituintes. Espera-se que a proposta contribua para suprir uma lacuna entre as experiências vivenciadas pelos alunos, no processo formação acadêmica e as experiências vividas no ambiente profissional e social. Nas considerações finais do artigo, procura-se refletir sobre os desafios para a implantação de um sistema de aprendizagem em ação no ensino em Administração.

\section{TEORIAS DA APRENDIZAGEM}

Os estudos em educação ${ }^{I}$ de adultos envolvem disciplinas como ciência política, economia, psicologia, antropologia, história e sociologia que desenvolvem diferenciadas perspectivas epistemológicas (CLARKE; BUTCHER, 2006). Foi em torno dos anos 1900 que a aprendizagem de adultos se tornou mais popular e emergiu em resposta a necessidades específicas, o que torna seu crescimento instável e irregular (MERRIAM; BROCKETT, 2007; KNOWLES, 1980).

Em 1970, Malcolm Knowles lançou nos Estados Unidos o conceito de que adultos e crianças aprendem de formas diferentes. Com base nas ideias de Lindeman (1926), Knowles (1975) introduziu em 1973 o termo andragogia, que abrange um conjunto de princípios que orientam a aprendizagem de adultos. Knowles, Holton e Swanson (20II) fazem uma análise sobre as críticas dos autores sobre a andragogia e destacam que Malcolm Knowles nunca pretendeu tornar a andragogia uma teoria da educação de adultos,

\footnotetext{
1 Neste artigo, o termo educação de adultos incorpora três significados: descreve os processos de aprendizagem de adultos, é um conjunto de atividades organizadas para alcançar os objetivos educacionais e também pode ser considerado um movimento ou campo da prática social (KNOWLES; HOLTON; SWANSON, 2011). Assim, o termo educação incorpora os processos de aprendizagem e o termo ensino de administração se refere aos processos de aprendizagem nas várias disciplinas do curso.
} 
mas um modelo transacional para compreender como os adultos aprendem em determinadas situações. Enquanto a pedagogia é definida por Kelly (2006) como a ciência de educar crianças e frequentemente usada como sinônimo de ensino, Beavers (2009) aponta a existência de diferenças claras entre as melhores práticas pedagógicas e as estratégias andragógicas, ratificando que a pedagogia não se aplica nos processos de aprendizagem de adultos.

Os principais pressupostos desenvolvidos por Lindeman (1926) e, posteriormente, por Knowles (1980) da aprendizagem de adultos revelam que: a) o aluno adulto e maduro é autodirecionado e aprende melhor por meio da ação; b) os adultos entram para a atividade educacional centrados na vida, em tarefas ou no problema de orientação para a aprendizagem e os motivadores mais potentes para adultos são internos, como a autoestima, reconhecimento, melhor qualidade de vida, autoconfiança e autorrealização; c) a experiência é a fonte mais rica para o adulto aprender; e d) os adultos têm a necessidade de aplicar os conhecimentos recém-adquiridos ou habilidades para suas circunstâncias imediatas e a aprendizagem deve ser vista como uma parceria entre docentes e alunos, cujas experiências devem ser usadas como um recurso.

O desenvolvimento das capacidades da aprendizagem autodirecionada talvez seja o principal objetivo da aprendizagem de adultos. Este autodirecionamento é geralmente definido em termos de comportamentos ou de atividades de aprendizagem explícita ao invés de procedimentos internos e disposições mentais (BROOKFIELD, 1986). Assim, a aprendizagem autodirecionada, desenvolvida por Knowles, focaliza a forma como os adultos podem aprender de maneira independente. Existem várias discussões sobre o que constitui a aprendizagem autodirecionada e pesquisadores como Knowles e Tough buscaram descrevê-la no contexto de seu processo sistemático (CRANTON, 2006).

Knowles (1975, p. I8) conceitua a aprendizagem autodirecionada como um processo em que "os indivíduos tomam iniciativa, com ou sem ajuda de outros, no diagnóstico de suas necessidades de aprendizagem, formulando objetivos de aprendizagem, identificando os recursos e materiais 
necessários, escolhendo e implementando as estratégias adequadas, assim como avaliando os resultados da aprendizagem".

Para Brookfield (1986), diversos estudos explicitam a complexidade em se definir a aprendizagem autodirecionada, pois alguns teóricos consideram o estado interno do aluno individual; já outros consideram aspectos do contexto social, por exemplo, os estudos de Brockett e Hiemstra (I99I), que enfatizam o processo de aprendizagem na perspectiva do aluno autodirecionado. Já as obras de Candy (I99I) abordam o tema do ponto de vista sociológico construtivista e distinguem entre o autodirecionamento como um "processo ou método de ensino" e como "um objetivo ou resultado".

Esse tipo de aprendizagem promove o desenvolvimento das pessoas em relação às suas competências emocionais, sociais, permitindo maior autonomia nas decisões, ou seja, uma liberdade com responsabilidade no controle de seus atos. Cranton (2006) ressalta que a aprendizagem autodirecionada contribui para a difusão dos pressupostos da teoria da aprendizagem transformadora. É uma teoria cuja perspectiva de significado pessoal de um adulto evolui e expande, tornando-o mais adaptável e capaz de obter benefícios com a experiência (PARKES, 200I).

A teoria da aprendizagem transformadora, desenvolvida por Jack Mezirow, no final dos anos 1960, objetiva transformações conscientes nos quadros de referência dos indivíduos, por intermédio da reflexão crítica sobre pressupostos construídos de modo acrítico. Pessoas com diferentes estilos de aprendizagem, estilos cognitivos e traços de personalidade podem assimilar e reconstruir as estruturas de referências de maneira distintas. Esta teoria envolve a aprendizagem em contextos mais estruturados ou menos estruturados, dirigindo-se à interseção entre o individual e o social, dimensões coexistentes e igualmente relevantes, já que os indivíduos fazem parte de uma sociedade (CRANTON, 2006).

Há três elementos principais da aprendizagem transformadora propostos por Mezirow (1990) as perspectivas de significado, os domínios de aprendizagem e os tipos de reflexão. As perspectivas de significado caracterizam a maneira como são vistas as coisas, as realidades construídas 
sob três perspectivas. A primeira é chamada de epistêmica e se refere àquilo que a pessoa sabe e como ela a conhece. Isso inclui os estilos de aprendizagem e preferências que não mudam facilmente. A segunda, sociolinguística, diz respeito às normas sociais e culturais. Estas são hábitos profundamente enraizados, que não são simples de se colocar em primeiro plano, para a consideração de uma forma, que poderia levar à transformação. A terceira é a perspectiva de significado psicológico (como a pessoa se vê como indivíduo), que pode ser resgatada em experiências da infância e não pode ser facilmente acessível ao seu eu consciente (MEZIROW, I990; 2000; CRANTON, 2006).

Com relação aos domínios de aprendizagem, Mezirow (199i) delimita três domínios: instrumental, comunicativo e emancipatório. O domínio da aprendizagem instrumental está relacionado ao conhecimento empírico do paradigma positivista, cuja ação é comandada por regras. O segundo domínio é o da aprendizagem comunicativa relacionado com o interesse cognitivo da prática, de modo a identificar como as pessoas aprendem como adultos. O terceiro, domínio da aprendizagem emancipatória, implica autorreflexão crítica, possivelmente levando a transformações dos esquemas ou de suas perspectivas de significado. Este último domínio envolve a forma como o indivíduo constrói a sua própria história, seus papéis e expectativas sociais. Os domínios de aprendizagem instrumental e comunicativa podem trabalhar juntos e interagir entre eles. Já o domínio emancipatório pode trabalhar em conjunto com qualquer um desses domínios, bem como pode trabalhar de forma independente (CRANTON, 2006; MEZIROW, 1990).

O terceiro elemento envolve os tipos de reflexão: conteúdo, processo e premissas. A reflexão do conteúdo se preocupa com "o que saber"; a reflexão do processo com o "como saber"; e a reflexão das premissas com o "por que se precisa saber". Tanto a reflexão de conteúdo, de processo e das premissas estão presentes nas três perspectivas de significado, como também nos três domínios de aprendizagem, como se observa no quadro I. (CRANTON, 2006; MEZIROW, I990; 199I).

London e Sessa (2006) ressaltam que para desenvolver as discussões em sala de aula que levem os alunos a mudanças em suas visões de mundo, 
por meio da reflexão crítica, é importante o uso do feedback como um determinante no processo de aprendizagem transformadora em grupo, uma vez que auxilia a conduzir o processo de transformação e retornar ao equilíbrio.

As teorias da aprendizagem servem de base para o desenvolvimento de um sistema de aprendizagem por meio da interação entre alunos, professores e o contexto da aprendizagem. A aprendizagem em ação amplia a visão dos atores envolvidos no proceso e contribui na redução ou eliminação da dicotomia teoria-prática, pensamento-ação como polos antagônicos, e os insere em um continuum para entender a aprendizagem no campo das organizações. 


\section{APRENDIZAGEM EM AÇÃO E ENSINO DE ADMINISTRAÇÃO}

A aprendizagem em ação foi introduzida por Revans, na década de 1940, e trilhou avanços nos Estados Unidos e no Reino Unido, com variações conceituais. Com o desenvolvimento de sua prática no ambiente organizacional, a expressão tornou-se, ao longo das décadas, coloquial no léxico, na área de gestão de pessoas (PEDLER, I997; WADDILL; BANKS; MARSH, 20IO).

Na concepção de Marquardt (1999), os problemas em tempo real, a reflexão sobre a relação das ações e resultados, o trabalho em equipe, o contexto de trabalho, as mudanças de perspectivas de significado e as experiências compartilhadas são algumas das premissas que fundamentam a aprendizagem em ação como uma abordagem para a resolução de problemas, além de contribuir para o desenvolvimento de competências por meio da aquisição de conhecimentos, troca de experiências e aprendizagem contínua (HANSON, 20IO; MARQUADT, 2009).

Em sua essência, a aprendizagem em ação é baseada na experiência de aprendizagem dos indivíduos. As pessoas potencializam a sua aprendizagem quando trabalham em um problema real e em tempo real (PEDLER, 1997; RAELIN, I997; REVANS, I998; WEINSTEIN, I995; DILWORTH; WILLIS, 2003). Ao discutir com seus pares um problema, ocorrem a reflexão e o desenvolvimento de ações para a busca de uma solução; neste momento, os indivíduos aprendem e desenvolvem a capacidade de adaptação. Todas as formas de aprendizagem em ação partilham de crenças/elementos, de pessoas reais resolvendo e agindo sobre os problemas reais em tempo real (PEDLER, I997; MARQUARDT, 2009). A aprendizagem em ação é uma forma de aprender não só baseada em ações, mas também por meio da dedicação do tempo necessário ao questionamento.

É difícil chegar a um consenso sobre o que realmente a aprendizagem em ação é em sua essência e, tal como descreve Pedler (1997), Revans sempre foi muito resistente em dar uma única definição para a aprendizagem em ação. Com essa atitude, Revans (1998) procurou preservar o seu aspecto filosófico, evitando tornar a aprendizagem em ação uma abordagem simples para $\mathrm{o}$ desenvolvimento gerencial. 
Buscando compreender as diferenças e as semelhanças, e alinhando as diferentes formas de pensamento dentro da arena de aprendizagem em ação, O'Neil e Marsick (2007) classificaram a aprendizagem em ação em quatro escolas, com a finalidade de compreender suas diferenças e semelhanças e alinhar as diferentes formas de pensamento dos autores.

Quadro 1 Escolas da aprendizagem em ação

\begin{tabular}{|c|c|c|c|c|}
\hline Escolas & Tácita & Científica & Experiencial & Reflexão crítica \\
\hline Teoria & $\begin{array}{l}\text { Aprendizagem } \\
\text { incidental }\end{array}$ & $\begin{array}{l}\text { Alfa, beta e } \\
\text { gama: } \\
L=P \& Q\end{array}$ & $\begin{array}{l}\text { Aprendizagem } \\
\text { baseada na } \\
\text { experiência }\end{array}$ & $\begin{array}{l}\text { Aprendizagem } \\
\text { por meio da } \\
\text { reflexão crítica }\end{array}$ \\
\hline Autores & $\begin{array}{l}\text { Dotlich e Noel; } \\
\text { Tichy }\end{array}$ & Revans; Boshyk & $\begin{array}{l}\text { McGill e Beaty; } \\
\text { Mumford }\end{array}$ & $\begin{array}{l}\text { Marsick; O'Neil; } \\
\text { Raelin }\end{array}$ \\
\hline
\end{tabular}

Fonte O’Neil e Marsick (2007).

Percebe-se que a escola tácita assume que a aprendizagem ocorre enquanto os participantes selecionados trabalham em conjunto, com as informações que são fornecidas pelos professores, no contexto da formação profissional. A escola cientifica está baseada no método científico para atingir os objetivos do grupo. A escola experiencial enxerga o ciclo de aprendizagem de Kolb como base teórica de aprendizagem. Por fim, a escola da reflexão crítica acredita que os participantes aprendem por meio de questionamentos e não apenas por meio de respostas prontas, o que possibilita a experimentação de novas maneiras de fazer as atividades, de pensar estrategicamente e trabalhar com diferentes pontos de vista (o'NeIL; MARSICK, 2007). A análise das escolas de aprendizagem em ação indica que a experiência e a reflexão crítica são mais adequadas ao contexto do ensino em Administração.

Smith (200I) ressalta que a aprendizagem em ação reconhece que os futuros administradores devem desenvolver autodirecionamento e autoconfiança, pois os programas de aprendizagem em ação sempre trabalham com grupos e os participantes são encorajados a discutir, compartilhar e reunir suas ambições e experiências e, portanto, a criarem algo diferente, um resultado coletivo, melhor do que poderiam ter sozinhos. 
Embora os estudiosos reconheçam a relevância da aprendizagem em ação, Yeo e Nation (2010) atentam que poucos têm enfatizado o impacto da ação sobre a orientação da aprendizagem. Ao contrário dos métodos tradicionais de sala de aula, que são, em grande parte, balizados pela aprendizagem mecânica e focados na memorização, a aprendizagem em ação, no campo do ensino, pode tornar o processo de aprendizagem mais significativo por meio da ação (SOFO; YEO; VILLAFA ÑE, 2OIO), uma vez que foca a reflexão experiencial como uma das bases de um sistema de aprendizagem. Entretanto, diversos estudos mostram o desequilíbrio entre aprendizagem e ação, que pode ser aprimorada com as práticas reflexivas e o exercício do feedback na busca de melhores soluções (CHO; EGAN, 20IO; SMITH, 200I). Ao olhar crítico de Schön (1983), é preciso romper com a ideia ortodoxa de uma única ciência e restabelecer as conexões entre ciência e arte, criando uma relação de complementaridade entre elas.

As mudanças em sala de aula estimulam os alunos a direcionarem e aprenderem por meio da utilização de fatos, relacionando suas ideias gerais para eventos específicos de sua vida, alerta Gutierrez (2002). Em concordância, Aktouf (2005) comenta que as práticas de ensino de administração não poderiam estar dissociadas do conteúdo.

Portanto, as condições do ambiente das universidades e as características da formação dos docentes são determinantes para o processo de aprendizagem dos alunos, pois ainda prevalece o papel passivo do aluno e a visão de que o professor é quem comanda o estudante (FREIRE, I98I).

Conforme Mintzberg e Gosling (2003), é na sala de aula que muitos administradores atuantes podem melhorar profundamente suas capacidades. No entender de Aktouf (2005), o ambiente da classe deve tornar todos os agentes envolvidos parceiros no processo de aprendizagem, favorecendo o desenvolvimento da inteligência, sensibilidade e reflexão. Para tanto, a educação em administração ainda necessita refletir sobre a difusão de práticas de ensino coerentes com as teorias de aprendizagem de adultos, que estimulem a troca de experiências, a articulação entre reflexão e ação, teoria e prática. As dimensões de um sistema de aprendizagem em ação, 
apresentadas na próxima seção, podem contribuir para a educação superior em administração, que possibilite dinamizar o ensino, estreitando a relação entre teoria e prática como também entre reflexão e ação, desencadeando um papel mais ativo entre os envolvidos no processo de aprendizagem. 


\section{DIMENSÕES DO SISTEMA DE APRENDIZAGEM EM AÇÃO NO} ENSINO DE ADMINISTRAÇÃO

A delimitação de um sistema de aprendizagem em ação no contexto do ensino de administração abrange cinco dimensões integradas e que podem contribuir para o planejamento e as ações da aprendizagem pelos gestores dos cursos e também pelos professores. A Figura i apresenta as dimensões que integram o sistema de aprendizagem em ação.

Figura 1 Sistema de Aprendizagem em ação no Ensino de Administração

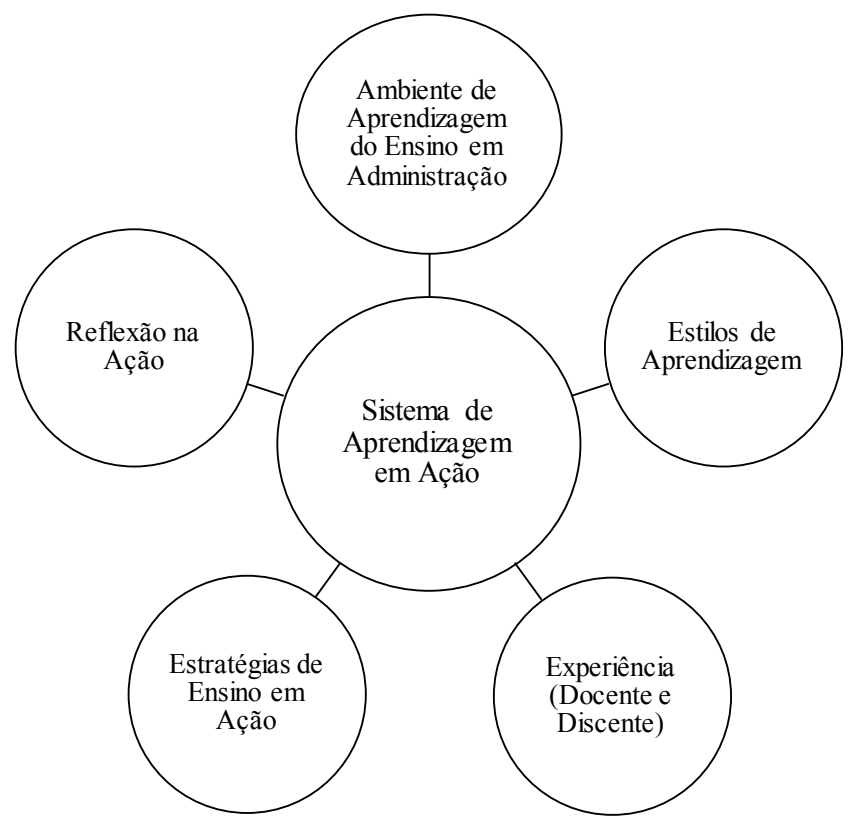

O contexto do ensino considera as influências internas e externas do ambiente da formação acadêmica em Administração, assim como as questões de espaço e tempo dos envolvidos no ambiente. Os estilos de aprendizagem caracterizam as formas de aprender dos alunos e a forma de ensinar dos professores. A reflexão em ação revela a necessidade de inserir no contexto do ensino o processo reflexivo, de modo a levar o aluno a refletir em ação sobre o conteúdo, o processo e as premissas. A experiência 
considera a necessidade de levar os agentes envolvidos no processo a refletirem sobre suas experiências para tornar o aprendizado significativo. As estratégias de ensino, balizadas pela aprendizagem em ação, estimulam o autodirecionamento, proporcionam o trabalho em equipe, incentivam a prática reflexiva, encorajam a troca de experiências e estimulam o debate e as discussões sobre os problemas e processos de tomadas de decisão, que podem promover mudanças nos seus quadros de referência e em suas perspectivas de significado. Os pressupostos de cada dimensão do sistema de aprendizagem em ação são abordados a seguir.

$1^{a}$ DIMENSÃO: AMBIENTE DA APRENDIZAGEM DO ENSINO EM ADMINISTRAÇÃO

O ensino superior em administração tem experimentado mudanças significativas, na última década, em que o ambiente de estudo fechado e a presença física dos alunos, necessários durante os estudos, não são mais as únicas formas de transmitir o conhecimento e promover a aprendizagem. Diversas universidades, principalmente em países europeus e nos Estados Unidos, já oferecem cursos em forma mista, ou seja, aulas presenciais e virtuais, permitindo que os alunos também possam aprender no seu contexto de trabalho. Essa forma ainda favorece a interação dos estudantes com outros países, o que ajuda a enriquecer suas experiências culturais e também promove uma relação interinstitucional mais forte. Porém, Oestreicher, Kuzma e Yen (2009) alertam que o uso dessas formas mistas no contexto do ensino indica a necessidade de analisar o domínio do professor com tais técnicas e também a maturidade do aluno para compreender e participar desses modelos de ensino mais modernos.

Merriam e Brocket (2007) consideram o ambiente de aprendizagem um conceito emergente na educação de adultos e destacam três dimensões, ainda pouco estudadas, que são o ambiente físico, o ambiente psicológico e o ambiente social. O ambiente físico envolve o espaço físico onde ocorre a aprendizagem. Apesar de os autores não contemplarem o ambiente virtual da aprendizagem, considera-se a necessidade de fazê-lo. O ambiente psicológico está relacionado à criação de um clima favorável de troca entre 
professores e alunos, procurando estabelecer um meio adequado para a vivência de experiências, que envolvem emoções como o medo, reflexões sobre suas dúvidas, para levá-los a se sentirem bem e compartilharem as suas experiências. O ambiente social focaliza os aspectos culturais, que envolvem o cenário do ensino-aprendizagem mediado pelo professor e considera aspectos como raça, gênero, além de princípios morais e sociais. Se utilizados de forma integrada, os elementos do ambiente da aprendizagem podem potencializar a aprendizagem de adultos.

$\mathrm{O}$ ambiente de aprendizagem do ensino em administração pode favorecer o uso de diferentes estratégias de ensino e proporcionar ao aluno um aprendizado por meio de situações de trabalho da vida real e da troca de experiências entre professor e aluno. Os estudos com estudantes universitários realizados por Chang, Lee, $\mathrm{Ng}$ e Jennings (200I) revelaram que a utilização da aprendizagem em ação, por parte dos professores, de maneira equivocada levou os alunos a alegarem que encontram casos até mais eficazes que seus próprios professores, comprovando o desconhecimento dos docentes de estratégias de ensino ativas, como, por exemplo, o método de casos. Jennings (2002) ressalta a necessidade dos professores identificarem os objetivos que mediarão o processo de ensino em sala de aula e utilizarem estratégias de ensino mais apropriadas.

Observa-se que o desejo de usar a internet tem levado gestores e professores de universidades a questionarem seus modelos tradicionais de ensino, pois percebem que os adultos aprendem melhor fazendo e recebendo feedback imediato. Assim, a andragogia pode ajudar as universidades a encontrar alternativas para alinhar melhor os seus programas as especificidades de seus alunos (BYRNE, 2002). Em qualquer programa de aprendizagem concebido para a internet, o processor pode utilizar as especificidades da aprendizagem autodirecionada no planejamento das atividades, pois como o ambiente virtual estimula os adultos a direcionarem o seu aprendizado. A internet é um meio de autoaprendizagem, que possibilita ao aluno a liberdade de busca de informações e fazer conexões com a informação que se adequa ao seu problema pessoal ou aos seus interesses. Uma dificuldade presente no ambiente virtual é justamente a falta de maturidade e de 
autodirecionamento dos alunos em seus processos de aprendizagem, o que limita a sua capacidade de aprender em ação.

As universidades passam por inovações tecnológicas, como a oferta de aulas online, utilizando-se do método de casos, adaptado para a sala de aula virtual para promover o pensamento crítico. Os alunos são desafiados a se envolverem em um ambiente virtual para aprender o conteúdo do curso por meio de trabalhos em equipes online e o professor se torna um facilitador para instruir os estudantes nesse novo formato em relação aos aspectos, por exemplo, da comunicação e interação (BROOKE, 2006). Com a utilização de estratégias de ensino mais adequadas ao contexto do ambiente virtual, pode-se desenvolver no aluno a responsabilidade pela sua própria aprendizagem e permitir que eles sejam desafiados a solucionar problemas em tempo real.

Destaca-se que os ambientes virtuais de aprendizagem não se restringem à educação a distância, pois eles também podem enriquecer as atividades em sala de aula por meio de tecnologias distintas e múltiplas abordagens de ensino. Ainda, ressalta-se que o ambiente virtual de aprendizagem não garante a eficácia por si só, uma vez que considera os fatores contextuais envolvidos, as habilidades do professor em utilizar estratégias e processos de ensino condizentes com a realidade dos alunos, para desenvolver o aprendizado deles e configurar as condições que potencializam os efeitos reais para enriquecer o campo educacional (DILLENBourG, SCHNEIDER E SYNTETA, 2002).

O uso de uma tecnologia no ambiente virtual de aprendizagem para a educação, segundo Beer, Slack e Armitt (2002), implica que o aluno vai ter experiências em um ambiente onde pode encontrar recursos para apoiar a sua aprendizagem. Desse modo, estratégias de ensino como os métodos de casos, a aprendizagem baseada em problemas, as simulações e os jogos empresariais proporcionam a interação entre ação e reflexão, estimulando o ambiente em que a aprendizagem pela troca de experiências significativas para docentes e discentes. Para isso, torna-se necessário conhecer os estilos de aprendizagem de cada agente envolvido no processo. 


\section{$2^{\mathrm{a}}$ DIMENSÃO: ESTILOS DE APRENDIZAGEM}

A teoria da aprendizagem experiencial (ELT) é assim chamada pelo seu idealizador Kolb (1984) por ser balizada pelos pressupostos das abordagens teóricas de John Dewey, Kurt Lewin e Jean Piaget bem como por enfatizar a geração do conhecimento por meio da transformação da experiência. Sharma e Kolb (2010) definem a aprendizagem como um processo de adaptação humana que envolve o indivíduo como um todo. A elt é aplicável não só em sala de aula da educação formal, mas em todas as áreas da vida.

Para aproximar aluno e professor, Kolb (1984) delimitou, a partir de autores basilares, pontos de convergência que fundamentaram uma perspectiva de aprendizagem com foco na experiência. García e Conde (2003) ressaltam que a teoria faz conexão com outras perspectivas de aprendizagem, como a andragogia de Knowles (1980), a aprendizagem transformadora de Mezirow (1991; 2000), os vínculos entre reflexão, ação e aprendizagem de Schön (1983) e a aprendizagem em ação.

Baseado na obra de Lewin - The Lewinian Experiential Learning Model - Kolb (1984) propôs quatro estágios adaptativos, o chamado ciclo de aprendizagem experiencial. A elt é uma visão dinâmica da aprendizagem com base em um ciclo de aprendizagem, composto pelas seguintes fases: a) experiência concreta: aprender experimentando; b) observação reflexiva: aprender observando; c) conceituação abstrata: aprender pensando; d) experimentação ativa: aprender fazendo. Para Kolb (1984), todo o processo de aprendizagem eficaz é cíclico. Este ciclo tem início com a Experiência Concreta (EC), depois com a Observação Reflexiva (OR), após assimilar a reflexão, ocorre a estruturação de fatos em teorias lógicas por meio da Conceituação Abstrata (CA) e, finalmente, as hipóteses são testadas em novas situações na Experimentação Ativa (EA).

A combinação dos quatro estágios de aprendizagem, dois a dois e em sequência, possibilitou a delimitação de quatro estilos individuais de aprendizagem e o desenvolvimento de um inventário de estilos de aprendizagem. Novos conhecimentos, habilidades e atitudes são alcançados por meio das relações entre os quatro modos de aprendizagem experiencial.

Como resultado da dupla combinação, surgem os quatro estilos de 
aprendizagem: Divergente $=\mathrm{EC}+\mathrm{OR} ;$ Assimilador $=\mathrm{CA}+\mathrm{OR} ;$ Convergente $=\mathrm{CA}+\mathrm{EA}$; e Acomodador $=\mathrm{EA}+\mathrm{EC}$. Kolb (1984) considera a aprendizagem vivencial um processo que envolve a aquisição e a transformação. A aquisição envolve o exercício da percepção, a decodificação de informações externas e o estabelecimento de vínculos com a sua vivência, juntamente com o repertório interno armazenado. Já o processo de transformação promove a geração de significados por meio da reflexão e da análise crítica. A aprendizagem envolve um ciclo de interações, no qual uma nova informação é experimentada, observada, refletida e conceituada.

Estilos de aprendizagem são, portanto, modos especializados de adaptação, reforçados pela escolha permanente de situações em que um estilo é bem-sucedido (коцв; коLв, 2005). Desde que um estilo específico de aprendizado represente uma preferência individual de apenas um ou dois dos quatro modos do ciclo de aprendizagem, sua eficácia é limitada às situações de aprendizagem, que envolvem a aquisição e a transformação.

A aprendizagem experiencial pode ser utilizada na prática educativa, uma vez que, para cada estilo de aprendizagem identificado, sejam sugeridas atividades e estratégias específicas de ensino, que apresentam uma visão holística de como as pessoas crescem e se desenvolvem (коцв; коLв, 2005).

Os estilos de aprendizagem também descrevem as diferenças individuais na aprendizagem baseada na preferência do aluno. Kolb (1984) assinala a importância do papel de um professor na identificação dos diferentes estilos de aprendizagem, além do estabelecimento de um processo de intervenção quando necessário, para potencializar a ocorrência da aprendizagem. Por considerar a experiência dos agentes envolvidos no processo de aprendizagem e os vários estilos de aprendizagem no ambiente do ensino, as dimensões do sistema de aprendizagem em ação podem ser potencializadas quando docentes e discentes reconhecem os seus estilos de aprendizagem e a experiência vivenciada no contexto fomenta uma integração entre ação, reflexão e aprendizagem. 


\section{3ª DIMENSÃO: EXPERIÊNCIA DO DOCENTE E DO DISCENTE}

Partindo do pressuposto de que a experiência é o conjunto de reações físicas, cognitivas e emocionais, que as pessoas experimentam quando interagem em um sistema social, o ambiente da educação formal é um local fundamental no desenvolvimento de experiências novas e significativas para o desenvolvimento pessoal e profissional, além de contribuir para a reflexão das experiências já vivenciadas. Experiência não é necessariamente o que se faz, mas, refere-se ao que acontece na vida das pessoas e traz algum significado para elas.

A aprendizagem de professores que atuam na educação superior envolve um conjunto de experiências significativas (ISAIA; MACIEL, 20II). Schön (I983) sugeriu que a capacidade de refletir na ação e sobre a ação, no processo de aprendizagem contínua, é uma característica definidora da prática profissional. Fomentar a troca de experiências no ambiente de aprendizagem é um desafio para os docentes, que pode ser viabilizado por meio do uso de estratégias de ensino, que fomentem a interação entre alunos e docentes, além de tornar a experiência de aprender significativa e transformadora. Dewey (I9ro) já dizia que ensinar e aprender estão interligados em um processo de aprendizagem. A vontade de ensinar do professor precisa ser compatibilizada com o interesse do aluno em querer aprender. Se esses interesses não forem compatibilizados, não há aprendizagem.

Hunt (20II) declara que toda decisão de um professor - o que ensinar como ensinar, e como avaliar a aprendizagem dos alunos - está enraizada na teoria. O professor pode não perceber, mas, ao fazer uma observação aparentemente elementar sobre a trama de uma obra ou a identificação de seu autor, ele tomou uma decisão teórica, uma decisão sobre o que é importante e vale a pena falar, bem como sobre o que seus alunos, provavelmente, ainda não sabem. Bondía (2002) afirmou que se costumava pensar sobre a educação do ponto de vista da relação entre a ciência e a técnica ou da relação entre teoria e prática. No primeiro caso, os educadores são considerados sujeitos técnicos, que aplicam o que foi pensado pela ciência; no segundo caso, os educadores passam a atuar como sujeitos críticos, que utilizam estratégias reflexivas distintas e consideram a educação sob uma perspectiva política. O autor 
sugere pensar a respeito da educação analisando, com base em experiência / sentido, uma possibilidade existencial e mais estética de reflexão.

Para Merriam (2008), quanto mais o professor compreender como os alunos aprendem, mais eficaz será a sua ação no ambiente de aprendizagem. Os alunos precisam ser estimulados pelos professores a aprenderem, não apenas cumprindo metas ou atingindo as notas estipuladas (BRUNER, 2009). Os educadores podem ir além da prática baseada em observações e estarem atentos para a identificação das condições essenciais para a aprendizagem (USHER, 2009).

A aprendizagem em ação de Revans (1998) enfatiza a ideia de que o aluno aprende em tempo real e sobre um problema real. Por isso, há necessidade de equilíbrio entre a ação e aprendizagem. É importante que o processo de aprendizagem seja diferenciado a ponto de as experiências vivenciadas na relação entre professores e alunos, de forma compartilhada para que o aluno auxilie no planejamento e operacionalização de um currículo (LINDEMAN, 1926). Um dos desafios dos docentes é subsidiar a difusão pelos discentes dos princípios da aprendizagem autodirecionada - necessidade de saber, autoconceito, experiências anteriores, prontidão para aprendizagem, orientação para aprendizagem e motivação para aprendizagem (KNOWLES, HOLSTON, SWANSON, 20II). Um meio de viabilizar a articulação teoria-prática, por meio da vivência de experiências que sejam significativas envolve a adoção de estratégias de ensino em ação.

\section{$4^{\mathrm{a}}$ DIMENSÃO: ESTRATÉGIAS DE ENSINO EM AÇÃO}

As estratégias de ensino adotadas pelos docentes impactam a maneira como os alunos aprendem. A articulação entre a teoria e os objetivos da disciplina com as necessidades dos estudantes é determinante para tornar o aprendizado significativo. Para tanto, ressalta-se a importância da difusão de estratégias de ensino que promovam uma maior relação entre a teoria e a prática, entre a reflexão e a ação, em um ambiente de aprendizagem que promova a formação em administração mais eficaz, desde que incorpore uma visão mais ampla sobre a atuação desse profissional no mundo do trabalho. 
Uma análise das práticas pedagógicas utilizadas na educação em administração indica a existência de quatro estratégias de ensino em ação (método de casos, aprendizagem baseada em problemas, simulações e jogos de empresas), como alternativas para conduzir o processo de aprendizagem articulado com a ação, e por apresentarem similaridades com os pressupostos da aprendizagem em ação.

$\mathrm{Na}$ difusão do método de casos, os estudantes aprendem no momento em que analisam as situações apresentadas e desenvolvem um plano de ação que propõe alternativas para a resolução de problemas, estimulando-os ao senso crítico e a criatividade (ROESCH, 2007). Sua conceituação tem sido confundida com o método de estudo de caso, uma estratégia de pesquisa utilizada para investigar fenômenos organizacionais. O método de casos ajuda a aperfeiçoar as habilidades analíticas dos alunos, que são desafiados pelos professores a utilizarem argumentos e análises para aprimorar tanto a capacidade de resolução de problemas como a capacidade de pensar e raciocinar com rigor (HAMMOND, 2002).

A Aprendizagem Baseada em Problemas (PBL), por outro lado, é uma estratégia de ensino centrada no estudante e surge com base em um problema real ou simulado, com o objetivo de estimular a solução do problema em questão, por meio do desenvolvimento do pensamento crítico, da capacidade de análise para a resolução de problemas, com o propósito de tornar o processo de aquisição de conhecimento mais consistente e duradouro sobre o tema pesquisado (GERTZMAN; KOLODNER, 1996). Nii e Chin (1996) afirmam que a PBL proporciona conhecimento aos alunos por meio de um processo de aprendizagem ativo, uma vez que melhora o raciocínio indutivo. A eficácia dessa estratégia está vinculada à flexibilidade, pois alunos e professores se envolvem em um processo de descoberta em conjunto. O professor tem o papel de estimular os alunos ao diálogo, à reflexão, à autonomia e ao aproveitamento das oportunidades de aprendizagem (LENNARTSSON, I996; KAUFMAN, 1998).

As simulações assumem a forma de um exercício em grupo que estimula os alunos na identificação, no estudo e no planejamento de novas iniciativas de negócios (JENNingS, 2000). Law e Kelton (199I) apresentam algumas de 
suas vantagens: a possibilidade da reaplicação dos experimentos, o que permite o teste de várias alternativas para o mesmo sistema; a avaliação de eventos vivenciados pelos agentes envolvidos no processo em um curto espaço de tempo; e a economicidade quando comparada às experiências no sistema real, pois estas podem acarretar consequências danosas ou irreparáveis. Ressalta-se que a simulação no campo educacional, por vezes, tem sido incapaz de criar uma tipologia, baseada na natureza da simulação, geralmente aceita (FEINSTEIN; CANNON, 20oI). Entretanto, um estudo realizado por Miles, Biggs e Schubert (1986) já evidenciava que os estudantes percebiam a simulação como uma estratégia poderosa de aprendizagem, que contribui na formação dos estudantes para agirem conscientemente no processo de tomada de decisão e em sistemas complexos (sHANNON, 1998).

Por fim, os jogos empresariais, impulsionados pelo surgimento dos computadores, melhoram o desempenho dos alunos, pois incentivam a visão holística e estratégica, além de tornar os alunos mais comprometidos, colaborativos e reflexivos (SAUAIA, 1995). Constata-se que as estratégias de ensino em ação resultam em um aprendizado mais ativo e transformador dos estudantes e fortalecem os pressupostos defendidos na proposição do sistema de aprendizagem em ação. A reflexão em ação é a ultima dimensão do sistema.

\section{$5^{\mathrm{a}}$ DIMENSÃO: A REFLEXÃO EM AÇÃO}

A reflexão é essencial na aprendizagem de adultos. O conceito de reflexão é complexo, pois não existe um consenso sobre o seu significado. A reflexão é a ponte entre a experiência e a aprendizagem, e envolve tanto a cognição como os sentimentos, além de promover uma conexão entre um conceito pessoal e outras formas de conhecimentos e experiências da pessoa (GRAY, 2007). Uma pessoa aprende quando "é capaz de refletir sobre suas ações e reorganizar, assim como reconstruir a experiência, por meio de um processo contínuo de reflexão - pensamento - como um meio de atuação" (ELKJAER, 200I, p. II3).

Para Daudelin (1996), a reflexão é um processo cognitivo pessoal, mas faz uma distinção entre a reflexão e a aprendizagem. Enquanto a reflexão 
está associada a um processo de pensar sobre uma experiência e considerar, de forma cuidadosa e persistente, o seu significado, baseado em inferências, a aprendizagem envolve a geração do significado de eventos passados ou atuais que orientam o comportamento futuro.

Dewey (200I) ressalta que os processos de feedback são as principais formas de reflexão. O conceito de Dewey sobre o pensamento reflexivo auxiliou os teóricos da aprendizagem de adultos. A característica comum da maioria das perspectivas teóricas da aprendizagem de adultos, por meio da experiência, é a relevância dada à integração da nova experiência e da experiência passada com o processo de reflexão. (RIGANO; EDWARDS, 1998).

O conceito de reflexão na ação foi proposto por Schön (1983) para ajudar as pessoas a lidarem com o conhecimento que, muitas vezes, é tácito e difícil de ser estruturado. A reflexão na ação é um processo que envolve a reflexão sobre as ações para descobrir como o ato de conhecer-na-ação contribuiu para um determinado resultado inesperado (sCHÖN, 2000). Um prático pode refletir sobre as normas tácitas e apreciações que são à base do julgamento, ou sobre as estratégias e teorias implícitas nos padrões de comportamento. Ele pode refletir sobre os sentimentos em uma situação que o levou a adotar um direcionamento específico para a ação ou sobre as ações direcionadas para um problema que tentou resolver, ou ainda sobre o papel construído por ele em um contexto institucional mais amplo (schöN, 1983).

O ciclo de ação e reflexão, quando desenvolvido de forma efetiva, levará a um processo de aprendizagem, uma vez que promove a integração entre o conhecimento e a prática por meio da reflexão (REYNOLDS, 1998). Daudelin (1996) descreve quatro estágios do processo reflexivo: a) articulação de um problema; b) análise do problema; c) formulação e experimentação de uma teoria para explicar o problema e d) ação ou decisão.

A reflexão também é usada de forma ambígua por alguns autores, pois há ocasiões em que o termo se refere à reflexão crítica. Outras vezes a reflexão é confundida tanto com a prática reflexiva como com a aprendizagem reflexiva. Entretanto, vários autores utilizam essas terminologias como semelhantes (MOON, 2004).

A reflexão sobre o processo de aprendizagem envolve o nível de 
consciência dos alunos sobre um processo de aprendizagem por meio da troca de experiências, seguida de uma fase de transformação e de uma área de reflexão, concebida como o processo de intervenção e de ligação entre as experiências, incorporando aspectos vinculados ao comportamento, às ideias, aos sentimentos e aos resultados, incluindo novas perspectivas sobre o compromisso da experiência para a ação (BOUD; KEOUGH; WALKER, I985). Para os autores, a reflexão envolve a integração entre pensamento e ação, uma oportunidade individual para modificar as estruturas de referência e de significados. No sistema de aprendizagem em ação, a reflexão atua como uma dimensão integradora para as outras dimensões, uma vez que é necessário considerar o contexto e os estilos de aprendizagem, utilizar as estratégias de ensino que incentivem a reflexão e que o processo fomente uma experiência significativa para os alunos e para os professores.

Para Moon (2004), existem fatores que articulam o processo de reflexão com o processo de aprendizagem. Alguns deles podem ser utilizados no planejamento das atividades de ensino e abrangem a aquisição de conhecimento por meio da ação, a construção da baseada na observações nas situações práticas, o processo de tomada de decisão e resolução de problemas e de revisão critica, além de resultados inesperados e esclarecimento e reconhecimento de que existe a necessidade de reflexão. A aprendizagem reflexiva ocorre quando um novo cenário e artefatos materiais torna o processo de aprendizagem.

O estímulo dos professores na busca de uma orientação mais reflexiva de seus alunos é pequeno. Como motivos para essa falta da prática reflexiva citam-se é o ritmo acelerado e as exigências do ambiente de trabalho que permitem pouco espaço para a reflexão. Esse contexto inclui fatores sociais, culturais, econômicos e políticos. A reflexão crítica suscita questões ligadas à moral, assim como de natureza técnica, com destaque para os as relações de poder presentes nas estruturas sociais e práticas, reconhecendo a experiência como um bem social e um fenômeno individual. Portanto, a reflexão crítica desafia normas existentes e o status quo, pois provoca inquietude e perturbações na pessoa para estimular a sua criticidade (REYNOLDS, I999; GRAY, 2007; FREIRE, I979). 


\section{CONSIDERAÇÕES FINAIS}

Este ensaio teórico propôs um sistema de aprendizagem em ação para o ensino de Administração, com o objetivo de refletir sobre a necessidade de pensar na aprendizagem, no curso de Administração, com base em uma perspectiva mais ampla, uma vez que as dimensões do sistema envolvem o ambiente de aprendizagem, os estilos de aprendizagem dos alunos e professores, o papel da experiência dos docentes e discentes na aprendizagem, a difusão de estratégias de ensino em ação e a necessidade de estimular os agentes envolvidos no processo de educação a refletirem em ação.

Para viabilizar a discussão em torno do sistema de aprendizagem em ação, foi preciso apresentar alguns pressupostos que envolvem a aprendizagem de adultos, com base nas perspectivas da andragogia, da aprendizagem autodirecionada e transformadora e da aprendizagem em ação.

A utilização do termo aprendizagem em ação objetivou minimizar a dicotomia entre teoria e prática, aproximar a relação professor-aluno no ambiente de aprendizagem, por meio da troca de experiências e do processo de reflexão em ação. Assim, compreender os estilos de aprendizagem dos professores e dos alunos pode auxiliar os agentes envolvidos nos processos de aprendizagem a se conscientizarem das características, que delimitam a sua forma de aprender e, com isso buscarem alternativas para fortalecer, repensar ou mudar as suas perspectivas de significado, potencializar os domínios de aprendizagem e fomentar a reflexão sobre o conteúdo, os processos e as premissas dos envolvidos nos processos de aprendizagem.

A implantação de um sistema de aprendizagem em ação em um curso de Administração demanda a necessidade de qualificação dos docentes envolvendo perspectivas teóricas da aprendizagem de adultos, além da criação de um ambiente físico, psicológico e social, que subsidie a mobilização de recursos pessoais, e do ambiente na operacionalização de disciplinas por parte dos docentes, em consonância com um projeto pedagógico do curso, que incorpore as perspectivas teóricas e as dimensões propostas neste artigo. Considera-se que as dimensões propostas no sistema 
podem integrar a agenda de discussões sobre os processos de aprendizagem dos alunos no curso de Administração. Por outro lado, a implantação do sistema de aprendizagem em ação no curso de Administração é balizada por vários desafios.

Um dos desafios envolve a revitalização de uma cultura marcada pela relação de dependência dos alunos aos professores e a estrutura do currículo do curso. O nível de autodirecionamento dos alunos é baixo, mesmo com o apoio dos professores, e isso pode implicar em baixo nível de reflexão sobre o que se aprende e como se aprende em sala de aula. Quando um professor desenvolve atividades que estimulam o pensamento reflexivo e demandam maior tempo para a realização das atividades, sofre criticas dos alunos que alegam, na maioria das vezes, falta de tempo para dedicação às atividades.

Outro desafio envolve o crescente número de alunos por turma. $\mathrm{Na}$ maioria das instituições, o número de alunos por turma chega a $60 \mathrm{e}$ até 70 alunos. Como promover estratégias de ensino em ação com um quantitativo tão grande de alunos por sala? Como adequar as estratégias de ensino aos vários estilos de aprendizagem dos alunos para tornar o processo de aprendizagem mais efetivo?

O estímulo à reflexão também se configura como um desafio, uma vez que os docentes se deparam com ementas com muito conteúdo e falta tempo para o desenvolvimento de atividades que promovam a reflexão na ação.

Também é necessário criar um ambiente físico, social e psicológico que estimule os alunos e professores a estabelecerem um processo de interação marcado pelo diálogo e pelo comprometimento com as atividades realizadas ao longo das disciplinas. Os recursos tecnológicos também são fundamentais nesse processo, uma vez que estimulam os alunos a interagir não só no ambiente presencial, mas também no virtual.

Por fim, as condições de trabalho dos docentes também se configuram como um desafio, pois a implantação do sistema de aprendizagem em ação demanda tempo e dedicação e muitos professores, sobretudo os das instituições particulares, são horistas e não têm muito tempo para atividades fora da sala de aula. 
O investimento em recursos tecnológicos e audiovisuais, a introdução de laboratórios voltados para a prática gerencial, a criação de centrais de casos para o ensino, a utilização de jogos e de simulações empresariais, a implantação de programas de capacitação para os docentes envolvendo perspectivas da aprendizagem orientadas para a ação, a aproximação das instituições com empresas públicas, privadas e do terceiro setor para discutir problemas vivenciados por empresários e gestores, a melhoria nas condições de trabalho dos docentes e a identificação dos estilos de aprendizagem dos alunos e dos docentes são ações que contribuirão para a introdução de um sistema de aprendizagem em ação para o ensino de Administração.

Espera-se que o artigo estimule o debate em torno da formação de administradores e auxilie os gestores dos cursos e os docentes a refletirem sobre as possibilidades de introdução das dimensões do sistema de aprendizagem em ação em seus cursos. Considera-se este artigo uma "semente" que foi plantada e algumas "árvores" já estão dando "frutos", que serão divulgados posteriormente para a comunidade científica. 


\section{REFERENNCIAS}

AKTOUF, O. Ensino de Administração: por uma pedagogia para a mudança. A administração e o conteúdo de ensino de Administração. O\&S, v. 12. n. 35, out./dez. 2005.

BEAVERS, A. Teachers As Learners: Implications Of Adult Education For Professional Development. Journal of College Teaching and Learning, v. 6, n. 7, 2009.

BEER, M.; SLACK, F.; ARMITT, G. Community building and virtual teamwork in an online learning environment. Hawaii International Conference on System Sciences. Computer Society, 2002.

BONDÍA, J. L. Notas sobre a experiência e o saber da experiência. Revista Brasileira de Educação, Rio de Janeiro, n. 19, p. 20-28, 2002.

BOUD, D.; KEOUGH, R.; WALKER, D. Reflection: Turning Experience into Learning, Kogan Page, London, 1985.

BROCKETT, R. G.; HIEMSTRA, R. Self-direction in adult learning: perspectives on theory, research and practice. London and New York: Routledge, 1991.

BROOKE, S. L. Using the case method to teach online classes: promoting socratic dialogue and critical thinking skills. International Journal of Teaching and Learning in Higher Education, v. 18, n. 2, 2006.

BROOKFIELD, S. D. Understanding and Facilitating Adult Learning. Jossey-Bass, San Francisco. 1986.

BRUNER, J. Culture, mind, and education. Chapter 11. In: ILLERIS, Knud (Ed). Contemporary theories of learning: learning theorists...in their own words. Routledge, 2009.

BYRNE, R. Web-based learning versus traditional management development methods. Singapore Management Review, v. 24, n. 2, 2002.

CANDY, P. C. Self-direction for lifelong learning. San Francisco: Jossey-Bass, 1991.

CHANG, J.; LEE, M.; NG, K.; JENNINGS, D. Strategic management and the case method: survey and evaluation in Hong Kong. Developments in business simulation and experiential learning, v. 28, 2001.

CHIEN, M. The Relationship between Self-Directed Learning Readiness and Organizational. Journal of American Academy of Business, Cambridge, mar. 2004.

CHO, Y.; EGAN, T. M. The state of the art of action learning research. Advances in Developing Human Resources, apr. 2010.

CLARKE, M; BUTCHER, D. Reconciling Hierarchy and Democracy: The Value of Management Learning. Management Learning, set. 2006.

CRANTON, P. Understanding and promoting transformative learning: a guide for educators of adults. San Francisco: Jossey-Bass Publishers. Second edition, 2006. 
DAUDELIN, M. W. Learning from experience through reflection. Organizational Dynamics, v. 24, n. 3, p. 36-49, 1996.

DEWEY, J. How we think. New York: D. C. Heath \& Co., Publishers, 1910.

DEWEY, J. The educational situation: as concerns the elementary school. Curriculum Studies, v. 33, n. 4, 2001.

DILLENBOURG, P.; SCHNEIDER, D.; SYNTETA, P. Virtual Learning Environments. In: PROCEEDINGS OF THE 3RD HELLENIC CONFERENCE INFORMATION \& COMMUNICATION TECHNOLOGIES IN EDUCATION. Anais... Kastaniotis Editions, Greece, 2002.

DILWORTH, R.L.; WILLIS, V.J. Action Learning: Images and Pathways. Krieger Publishing Company. Malabar, FL, 2003.

ELKJAER, B. Em busca de uma teoria de aprendizagem social. In: EASTERBY-SMITH, M.; BURGOYNE, J.; ARAUJO, L. (Coord.). Aprendizagem organizacional e organização de aprendizagem: desenvolvimento na teoria e na prática. São Paulo: Atlas, 2001.

FEINSTEIN, A.; CANNON, H. Fidelity, verifiability, and validity of simulation: Constructs for Evaluation. Developments in Business Simulation and Experiential Learning, 2001.

FREIRE, P. Educação e Mudança. Rio de Janeiro: Paz e Terra, 1979.

FREIRE, P. Pedagogia do oprimido. 10.ed. Rio de Janeiro: Paz e Terra, 1981.

GERTZMAN, A.; KOLODNER, J. L. A case study of Problem-Based Learning in a middle school science class: lessons learned. Edu Tech Institute. Georgia Institute of Technology. Atlanta, 1996.

GRAY, D. E. Facilitating Management Learning: Developing Critical Reflection Through Reflective tools. Management Learning, nov. 2007.

GUTIERREZ, R. Change in classroom relations: An attempt that signals some difficulties. Journal of Management Education, v. 26, n. 5, oct. 2002.

HAMMOND, J. S. Learning by the case method. Harvard Business School. Rev. April 16, 2002.

HANSON, K. R. Action Learning Strategies on Continuous Improvement Efforts in Elementary Schools. 2010. Thesis (Doctor of Education in Organizational Leadership). University of La Verne, La Verne, California, 2010.

HUNT, T. J. An epistemological approach to literature: creating a paradigm for literary study in the ib language A1 classroom. 2011. Thesis (In partial fulfillment of the requirements For the Degree of Master of Arts Colorado State University Fort Collins), Colorado Spring, 2011.

ISAIA, S. M. de A.; MACIEL, A. M. de R. Comunidades de práticas pedagógicas universitárias em ação: Construindo a aprendizagem docente. Imagens da Educação, v. 1, n. 1, p. 37-47, 2011. 
JENNINGS, D. Strategic management: an evaluation of the use of three learning methods. Developments in Business Simulation \& Experiential Learning, v. 27, 2000.

JENNINGS, D. Strategic management: an evaluation of the use of three learning methods. The Journal of Management Development, v. 21, n. 9, 2002.

KAUFMAN, D. M. Problem-Based Learning: Using cases to teach about how to deal with ethical problems. NCEHR, v. 8, n. 2, 1998.

KELLY, Michelle H. Teach an Old Dog New Tricks: Training Techniques for the Adult Learner. Professional Safety, ago. 2006.

KNOWLES, M. S.; HOLTON, E. F.; SWANSON, R. A. The adult learner. The definitive classic in adult education and human resource development. Elsevier, Seventh Edition, 2011.

KNOWLES, M. S. Self-directed learning. New York: Association Press, 1975.

KNOWLES, M. S. The modern practice of adult education: from pedagogy to andragogy. Cambridge Adult Education, 1980.

KOLB, A. Y.; KOLB, D. A. Learning Styles and Learning Spaces: Enhancing Experiential Learning in Higher Education. Academy of Management Learning \& Education, v. 4, n. 2, p. 193-212, 2005.

KOLB, D. A. Experimental learning: Experience as the source of learning and development. New Jersey: Prentice-Hall, Englewood Cliffs, 1984.

LAW, A. M.; KELTON, W. D. Simulation, modeling \& analysis. Second Edition. New York: McGraw-Hill, 1991.

LENNARTSSON, B. Experiences from direct application of the PBL method in industrial training and organization development. Department of Computer and Information Science Linköping, 1996.

LINDEMAN, E. C. The meaning of Adult Education. New Republic, INC. New York, 1926.

LONDON, M.; SESSA, V. I. Group Feedback for Continuous Learning. Human Resource Development Review, v. 5, n. 3, p. 303-329, set. 2006.

MARQUARDT, M. Action Learning in action: Transforming problems and people for world-class organizational learning. CA: Davies-Black, 1999.

MARQUARDT, M. Optimizing the power of action learning: Solving problems and building leaders in real time. Davies-Black. London, 2004.

MARQUARDT, M. Optimizing the power of action learning. Solving problems and building leaders in real time. Davies-Black. Boston; London, 2009.

MERRIAM, S. B. Third update on adult learning theory. Adult learning theory for the twenty-first century. Capítulo 9, nº 119. San Francisco: Jossey-Bass, 2008.

MERRIAM, S. B.; BROCKETT, R. G. The profession and practice of adult learning: an introduction. San Francisco: Jossey-Bass, 2007. 
MEZIROW, J.; ASSOCIATES. Fostering critical reflection in adulthood: A guide to transformative and emancipatory learning. San Francisco, California. Jossey-Bass, 1990. MEZIROW, J. Transformative Dimensions of Adult Learning. San Francisco: Jossey Bass, 1991.

MEZIROW, J. Learning to think like an adult: Core concepts of transformation theory. In: MEZIROW, J. \& Associates. (Eds.) Learning as transformation: Critical perspectives on a theory in progress. San Francisco, CA. Jossey-Bass, 2000.

MILES, G. M.; BIGGS, W.; SCHUBERT, J. Student perceptions of skill acquisition through cases and a general management simulation. Simulation \& Games: An International Journal, v. 17, n. 1, p. 7-24, 1986.

MINTZBERG, H.; GOSLING, J. Educando administradores além das fronteiras. RAE, v. 43, n. 2, 2003.

MOON, J. A. A handbook of reflective and experiential learning: Theory and practice. Routledge Falmer, 2004.

NII, L. J.; CHIN, A. Comparative trial of Problem-Based Learning versus Didactic Lectures on clerkship performance. American Journal of Pharmaceutical Education, v. 60, Summer, 1996.

O’NEIL, J.; MARSICK, V. J. Understanding action learning: Theory into practice. AMACON, 2007.

OESTREICHER, K.; KUZMA, J.; YEN, D. The virtual university and avatar technology: e-learning through future technology. Worcester Business School, University of Worcester, 2009.

PARKES, D. About Adult Education - Transformative Learning. Journal of Workplace Learning, v. 13, n. 4, p. 182-183, 2001.

PEDLER, M. What do you mean by Action Learning? A story and three interpretations. In: PEDLER, M. (Ed.) Action learning in practice (3ed.). Aldershot: Gower, 1997, p. 61-75. RAELIN, J.A. Model of work based-learning. Organization Science, v.8, n.6, p. 563-578, 1997.

REVANS, R. ABC of Action Learning: Empowering managers to act and to learn from action. Lemos \& Crane, n. 6, London, 1998.

REYNOLDS, M. Reflection and critical reflection in management learning. Management Learning, v. 20, n. 2, p. 183-200, 1998.

REYNOLDS, M. Critical reflection and management education: Rehabilitating less hierarchical approaches. Journal of Management Education, v. 23 n. 5, out. 1999.

RIGANO, D.; EDWARDS, J. Incorporating reflection into work practice: A case study. Management Learning, dec. 1998.

ROESCH, S. M. A. Casos de ensino em administração: Notas sobre a construção de casos para ensino. RAC, v. 11, n. 2, abr/jun. 2007. 
SAUAIA, A. C. A. Satisfação e aprendizagem em jogos de empresas: contribuições para a educação gerencial. Tese (Doutorado em Administração). 273 págs. Faculdade de Economia, Administração e Contabilidade da Universidade de São Paulo. São Paulo, 1995.

SCHÖN, D. A. The Reflective Practitioner: how professionals think in action. London: Temple Smith, 1983.

GARCÍA, A. V. M.; CONDE, M. J. R. Estilos de Aprendizaje y Educación Superior. Análisis discriminante en función del tipo de estudios. Enseñanza, v. 21, n. 1, p. 77-97, 2003.

SCHÖN, D. A. Educando o profissional reflexivo: um novo design para o ensino e a aprendizagem. Porto Alegre: Artes Médicas Sul, 2000.

SHANNON, R. E. Introduction to the Art and Science of Simulation. Winter Simulation Conference. IEEE Computer Society Press Los Alamitos, CA, USA, 1998.

SHARMA, G.; KOLB, D. A. The Learning Flexibility Index: Assessing Contextual Flexibility in Learning Style. Department of Organizational Behavior Case Western Reserve University Working Paper Series, jan. 2010.

SMITH, P. A. C. Action Learning and Reflective Practice in Project environments that are related to leadership development. Management Learning, 2001.

SOFO, F.; YEO, R. K.; VILLAFAÑE, J. Optimizing the learning in action learning: reflective questions, levels of learning and coaching. Advances in Developing Human Resources, 2010.

USHER, R. Experience, pedagogy and social practices. Chapter 12. In: ILLERIS, Knud (Ed). Contemporary theories of learning: learning theorists...in their own words. Routledge, 2009.

WADDILL, D.; BANKS, S.; MARSH, C. The Future of Action Learning. Advances in Developing Human Resources, 2010.

WEINSTEIN, K. Action learning: a journey in discovery and development. London: Harper Collins Publishers, 1995.

YEO, R. K.; NATION, U. E. Optimizing the action in action learning: urgent problems, diversified group membership and commitment to action. Advances in Developing Human Resources, 2010. 


\section{DADOS DOS AUTORES}

ANIELSON BARBOSA DA SILVA` anielson@pq.cnpq.br

Doutor em Engenharia de Produção pela UFSC

Instituição de vinculação: Universidade Federal da Paraíba

João Pessoa/PB - Brasil

Áreas de interesse em pesquisa: Aprendizagem Gerencial e Organizacional,

Gestão por Competências, Comportamento Organizacional e Gestão Pública.

^ Endereço: Universidade Federal da Paraíba - Centro de Ciências Sociais Aplicadas -

Programa de Pós-Graduação em Administração

Campus Universitário Castelo Branco João Pessoa/PB 58059-900

THALES BATISTA DE LIMA thalesufpb@gmail.com

Mestre em Administração pela UFPB

Instituição de vinculação: Universidade Federal da Paraíba

João Pessoa/PB - Brasil

Áreas de interesse em pesquisa: Organizações e Recursos Humanos.

ANA LÚCIA BAGGIO SONAGLIO anabaggiosonaglio@hotmail.com Mestre em Administração pela UNIVALI

Instituição de vinculação: UNIVALI

Biguaçu/SC - Brasil

Áreas de interesse em pesquisa: Administração de Empresas.

CHRISTIANE KLEINÜBING GODOI chriskg@univali.br

Doutora em Engenharia de Produção pela UFSC

Instituição de vinculação: Universidade do Vale do Itajaí

Biguaçu/SC - Brasil

Áreas de interesse em pesquisa: Metodologia de pesquisa qualitativa e análise do discurso.

$\dagger$ O presente trabalho foi beneficiário de auxílio financeiro da CAPEs, por meio do Edital Pró-Administração. 